\section{Die Temperaturabhängigkeit \\ der Dielektrizitätskonstanten von Seignettesalz orthogonal zur ferroelektrischen Achse}

\author{
K. Forsch und H. E. Müser
}

Institut für Experimentalphysik II der Universität Saarbrücken (Z. Naturforsch. 23 a, 1231 [1968] ; eingegangen am 28. Mai 1968)

Vor etwa 30 Jahren wurden die Dielektrizitätskonstanten von Seignettesalz orthogonal zur ferroelektrischen $a$-Achse von Mueller ${ }^{1}$, Hablützel ${ }^{2}$ und Mason ${ }^{3}$ zum ersten Mal etwas genauer untersucht. Die Ergebnisse dieser drei Autoren stimmen nicht besonders gut miteinander überein. Wir haben deshalb mit den heute zur Verfügung stehenden genaueren Meßvorrichtungen Absolutwerte und Temperaturabhängigkeit der beiden Dielektrizitätskonstanten erneut bestimmt.

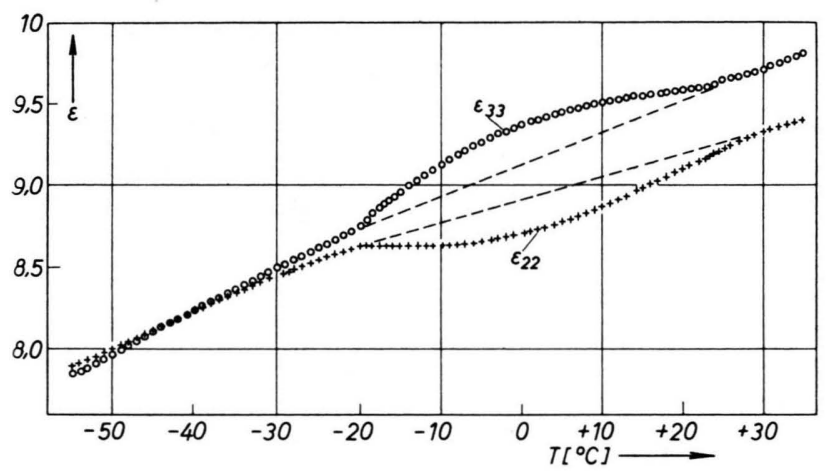

Abb. 1. Die Temperaturabhängigkeit der relativen Dielektrizitätskonstanten $\varepsilon_{22}$ in $b$ - und $\varepsilon_{33}$ in $c$-Richtung von Seignettesalz. Meßfrequenz: $1 \mathrm{kHz}$. Meßfeldstärke ca. $5 \mathrm{~V} / \mathrm{mm}$. Relative Luftfeuchtigkeit: $12 \%$.

Abb. 1 zeigt die Temperaturabhängigkeit der relativen Dielektrizitätskonstanten $\varepsilon_{22}$ und $\varepsilon_{33}$ in Richtung der kristallographischen $b$ - bzw. $c$-Achse, die aus Kapazitätsmessungen bei $1 \mathrm{kHz}$ mit einer General-RadioBrücke (Typ 1620-A) ermittelt wurden. Zwischen -55 und $+35{ }^{\circ} \mathrm{C}$ wachsen $\varepsilon_{22}$ und $\varepsilon_{33}$ monoton von etwa 7,9 auf 9,4 bzw. 9,8 an. Obwohl die Streuung der Meßwerte äußerst gering und die Reproduzierbarkeit bei vorgegebener Probe sehr gut war, beträgt die Unsicherheit der Absolutwerte etwa 2\%. Dies ist hauptsächlich auf Ungenauigkeiten bei der Bestimmung der Probengeometrie und des Streufeldbeitrages, zum Teil vielleicht auch auf echte Schwankungen der Stoffeigenschaften von Probe zu Probe, zurückzuführen.

1 H. Mueller, Phys. Rev. 47, 175 [1935].

2 J. Hablützel, Helv. Phys. Acta 12, 489 [1939].

3 W. P. MAson, Piezoelectric Crystals and Their Application to Ultrasonics, van Nostrand, New York 1950.
Im ferroelektrischen Temperaturbereich zeigen beide Kurven charakteristische Abweichungen von der zwischen den parelektrischen Bereichen interpolierten Temperaturabhängigkeit. Der interpolierte Verlauf ist in der Abbildung durch gestrichelte Geraden angedeutet. Eine Analyse der Abweichungen ergab, daß sie dem Quadrat der spontanen Polarisation proportional sind.

Zur Einordnung dieses Sachverhaltes in das bereits bekannte Erscheinungsbild von Seignettesalz eignet sich die freie elastische Enthalpie $G_{1}$ besonders gut ${ }^{4}$. Die unabhängigen Variablen dieser thermodynamischen $\mathrm{Zu}$ standsfunktion sind die mechanischen Spannungen, die dielektrischen Verschiebungen $D_{1}, D_{2}$ und $D_{3}$ in $a$-, $b$ - bzw. $c$-Richtung und die Temperatur. An dieser Stelle sollen der Einfachheit halber nur die Terme von $G_{1}$ betrachtet werden, die $D_{1}{ }^{4}, D_{2}{ }^{2}$ oder $D_{3}{ }^{2}$ enthalten: $G_{1}=\ldots+\frac{1}{4} B_{11} D_{1}^{4}+\frac{1}{2} \beta_{22} D_{2}^{2}+\frac{1}{2} \beta_{33} D_{3}^{2}+\ldots$.

$B_{11}$ ist die Sättigungskonstante, die von Mueller zur phänomenologischen Beschreibung von Seignettesalz eingeführt wurde $^{5}$. Die Dielektrizitätskonstanten $\beta_{22}$ und $\beta_{33}$ verknüpfen die dielektrischen Verschiebungen $D_{2}$ und $D_{3}$ mit den zugehörigen Feldstärken. Sie sind wegen der im ferroelektrischen Bereich auftretenden Abweichungen der Dielektrizitätskonstanten vom interpolierten Verlauf als Funktionen von $D_{1}$ anzusehen, und zwar gilt

$$
\begin{aligned}
& \beta_{22}=\beta_{22}{ }^{0}+B_{12} D_{1}{ }^{2}, \\
& \beta_{33}=\beta_{33}{ }^{0}+B_{13} D_{1}{ }^{2} .
\end{aligned}
$$

$\beta_{22}{ }^{0}$ und $\beta_{33}{ }^{0}$ sind die von $D_{1}$ unabhängigen Anteile der Dielektrizitätsmoduln mit „normaler“ Temperaturabhängigkeit. $B_{12}$ und $B_{13}$ sind Konstanten, deren Bedeutung durch Einsetzen von Gl. (2) in (1) zu ersehen ist. Man erhält statt der in Gl. (1) aufgeführten Glieder

$$
\begin{aligned}
G_{1}=\ldots & +\frac{1}{2} \beta_{22}{ }^{0} D_{2}{ }^{2}+\frac{1}{2} \beta_{33}{ }^{0} D_{3}{ }^{2}+\frac{1}{4} B_{11} D_{1}{ }^{4} \\
& +\frac{1}{2} B_{12} D_{1}^{2} D_{2}{ }^{2}+\frac{1}{2} B_{13} D_{1}{ }^{2} D_{3}{ }^{2}+\ldots .
\end{aligned}
$$

Zur vollständigen Beschreibung des dielektrischen Verhaltens von Seignettesalz müssen also in der freien elastischen Enthalpie noch zwei zusätzliche Glieder vierter Ordnung berücksichtigt werden. Die Beträge der Koeffizienten $B_{12}$ und $B_{13}$ haben die gleiche Größenordnung wie die Sättigungskonstante $B_{11}$ :

$$
\begin{aligned}
& B_{11}=59 \cdot 10^{12} \mathrm{Vm}^{5} / \mathrm{C}^{3}, \\
& B_{12}=33 \cdot 10^{12} \mathrm{Vm}^{5} / \mathrm{C}^{3}, \\
& B_{13}=-35 \cdot 10^{12} \mathrm{Vm}^{5} / \mathrm{C}^{3} .
\end{aligned}
$$

Der hier für $B_{11}$ angegebene Wert ist aus vorliegenden Ergebnissen ${ }^{6,7}$ gemittelt worden.

${ }^{4}$ H. E. Müser, Ann. Phys. 14, 176 [1964].

5 H. Mueller, Phys. Rev. 58, 565 [1940].

${ }^{6}$ H. E. Müser, Z. Angew. Phys. 12, 301 [1960].

7 G. Sснмidt, Phys. Stat. Sol. 8, 41 [1965]. 